\title{
ANÁLISE BIOMECÂNICA DO PROCESSAMENTO DA BANANA EM \\ UMA COOPERATIVA CATARINENSE
}

\section{BIOMECHANICAL ANALYSIS OF BANANA PROCESSING IN A COOPERATIVE INDUSTRY LOCATED IN SANTA CATARINA}

Giselle Schmidt A D Merinoํㅜㄹ D.Sc

Diego Luiz de Mattos², M.Sc

Lincoln da Silva ${ }^{3}$, M.Sc

Bruno Maia Guimaraes ${ }^{4}$, D.Sc

Noe Gomes Borges Junior ${ }^{5}$, PhD.Sc

Susana Cristina Domenech ${ }^{6}$, D.Sc

Eugenio A D Merino ${ }^{7}$, PhD.Sc

(1) Universidade Federal de Santa Catarina e-mail: gisellemerino@gmail.com

(2) Universidade Federal de Santa Catarina e-mail:diegoclerigo@yahoo.com.br

(3) Universidade Federal de Santa Catarina e-mail: lincoln_floripa@yahoo.com.br

(4) Instituto Federal de Santa Catatina e-mail:bmguimaraes@gmail.com

(5) Universidade do Estado de Santa Catarina e-mail:nborgesjr@gmail.com

(6) Universidade do Estado de Santa Catarina e-mail: scdomenech@gmail.com

(7) Universidade Federal de Santa Catarina e-mail:eugenio.merino@ufsc.br 
$16^{\circ}$ USIHC - Congresso Internacional de Ergonomia e Usabilidade de Interfaces Humano Computador

CINAHPA | 2017 - Congresso Internacional de Ambientes Hipermídia para Aprendizagem.

\section{Ergonomia; Bananicultura; Biomecânica.}

Este estudo teve por fim identificar os riscos físicos inerentes a atividade de cultivo e processamento da banana em uma cooperativa Catarinense. Os métodos utilizados foram a dinamometria manual e a captura de movimento. Os resultados apresentaram, em relação à análise do movimento do ombro direito, uma média dos ângulos de abdução de $32,38^{\circ} \pm 13,88$ e flexão de $33,71^{\circ} \pm 22,66$, dados compatíveis com situações que incorrem em risco de lesão. Em relação a dinamometria de preensão manual, apesar do risco demonstrado inerente ao tipo de movimentação nos membros superiores, a força isométrica de preensão manual obteve valores acima da média nacional para homens (Membro Dominante: 44,2 $\pm 8,9$ e Membro Não Dominante: $40,5 \pm 8,5)$. As conclusões indicam que a atividade da despenca de bananas apresenta riscos de distúrbios osteomusculares no ombro direito, nos punhos direito e esquerdo dos trabalhadores devido às posturas adotadas e a repetitividade.

\section{Ergonomics; Banana cultivation; Biomechanics}

This study aimed to identify the physical the physical risks inherent to banana cultivation and processing activity in a Santa Catarina cooperative. The methods used were manual dynamometry and motion capture. The results showed a mean abduction angles of $32.38 \pm 13.88$ and a bending of $33.71 \pm 22.66$ in the right shoulder, data compatible with situations that are at risk of injury. Regarding hand grip dynamometry, despite the demonstrated risk inherent to the type of movement in the upper limbs, the manual grip isometric force obtained values above the national average for men (Dominant Member: $44.2 \pm 8.9$ and Member Non-dominant: $40.5 \pm 8.5$. The results of the research showed that the activity of banana processing presents risks of musculoskeletal disorders in the right shoulder and in the right and left wrists of the workers due to the adopted postures and the repetitiveness.

\section{Introdução}

O cooperativismo é uma importante vertente econômica no Brasil. Em Santa Catarina, o cooperativismo cresceu $12,96 \%$ no ano de 2015 , alcançando uma receita bruta de 27,5 bilhões, de acordo com a Organização das Cooperativas do Estado de Santa Catarina (Ocesc). No estado, O agronegócio representa $64 \%$ da movimentação das cooperativas, tendo no período uma alta na receita bruta na ordem de $12 \%$, correspondendo a $\mathrm{R} \$ 17,3$ bilhões. Entretítulos e Subtítulos

Umas das atividades registradas pelo cooperativismo catarinense é o cultivo da Banana. A banana (Musa spp.), pertencente à família Musaceae, é considerada a fruta mais produzida e a mais consumida no mundo com uma produção de 106,5 milhões de toneladas, sendo explorada na maioria dos países tropicais e se destaca como uma das principais fontes de arrecadação e geração de emprego e renda (CEPEA, 2016).
Na região Sul do Brasil, em especial no Estado de Santa Catarina, a bananicultura é uma atividade agrícola de grande importância social devido a geração de emprego e renda aos trabalhadores. Em termos sociais, é explorada por cerca de $25 \mathrm{mil}$ produtores rurais, sendo que seis mil têm na bananicultura sua principal atividade de renda.

Dada s importância e relevância do cooperativismo agropecuário, é importante salientar que, de maneira geral, as atividades rurais, por ocasião de sua natureza física, têm consequências sobre a saúde do trabalhador. Segundo dados da Previdência Social, foram emitidos 12.817 auxílios acidentes de trabalho para trabalhadores rurais no ano de 2014, enquanto que os custos sucedidos da emissão desses benefícios foram de $\mathrm{R} \$ 4.637 .000$ (Brasil, 2015). O trabalho neste setor exige posturas inadequadas, manuseio de cargas e movimentos repetitivos, fatores reconhecidamente de risco para doenças musculoesqueléticas (Fathallah, 2010). 


\section{$16^{\circ}$ \\ ERGODESIGN USIHC CINAHPA}

Em relação às doenças musculoequeléticas, a despenca da banana pode apresentar importante risco postural, principalmente nos membros superiores (TAKAYAMA et al, 2015).

A convergência desses fatores delineados pela representatividade do cooperativismo agropecuário nacional e do estado de Santa Catarina e, mais especificamente, da produção de banana, a natureza da realização das tarefas, as condições de trabalho que podem culminar no adoecimento do trabalhador e afastamento de suas atividades por lesões de natureza musculoesquelética, apontam para uma lacuna de conhecimentos que requer a compreensão das consequências físicas do trabalho rural. Portanto o problema deste estudo pode ser definido através do questionamento: Quais são os riscos físicos do trabalho em uma cooperativa de agricultura familiar de bananas, relacionados aos membros superiores?

Diante do exposto, esta pesquisa teve como objetivo identificar os riscos de lesões musculoesqueléticas dos membros superiores da atividade citada de maneira que o conjunto de informações possibilite o entendimento inequívoco da situação, visando futuras intervenções ergonômicas, com recorte na atividade da despenca de bananas.

\section{Materiais e Métodos}

\subsection{Caracterização Geral}

O estudo foi realizado em uma cooperativa produtora de bananas localizada no município de Corupá (26 $\left.25^{\prime} 30^{\prime \prime} \mathrm{S} 49^{\circ} 14^{\prime} 34^{\prime \prime} \mathrm{O}\right)$, região Norte do Estado de Santa Catarina (Figura 01). A

Cooperativa atua há mais de 20 anos e emprega mais de 60 associados, todos agricultores familiares. Destes, apenas 15 trabalhadores atuam diretamente no beneficiamento da banana, sendo o restante responsável pelo cultivo, corte e transporte. No local são produzidas três variedades de banana: prata, caturra e maçã. $16^{\circ}$ Ergodesign - Congresso Internacional de Ergonomia e Usabilidade de Interfaces Humano Tecnológica: Produto, Informações Ambientes Construídos e Transporte

$16^{\circ}$ USIHC - Congresso Internacional de Ergonomia e Usabilidade de Interfaces Humano Computador

CINAHPA | 2017 - Congresso Internacional de Ambientes Hipermídia para Aprendizagem.

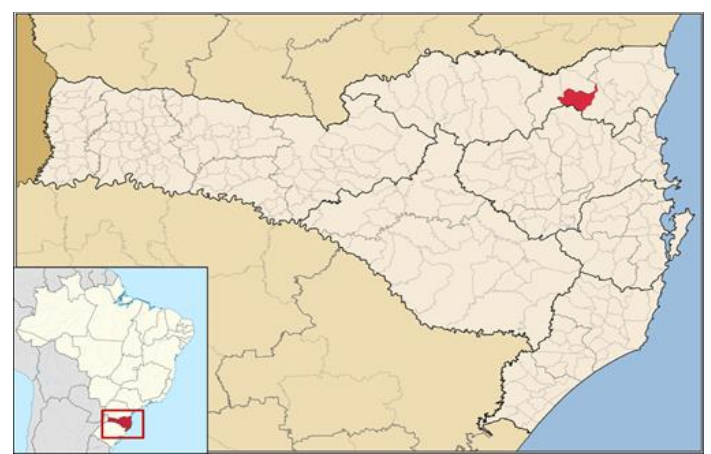

Figura 01: Localização do Município de Corupá, no estado de Santa Catarina.

Na cooperativa do estudo, para a realização de todas as etapas do fluxo produtivo a jornada de trabalho é intensa e variável sendo influenciada diretamente pela demanda. A produção é comercializada principalmente para o mercado da cidade de Pato Branco (PR). Não existe um treinamento formal sobre as tarefas que precisam ser executadas e sobre práticas de saúde e segurança do trabalho na atividade. Os trabalhadores aprendem a tarefa no dia a dia com outros agricultores mais experientes.

A amostra foi composta por 3 trabalhadores do gênero masculino que aceitaram participar da pesquisa e atenderam aos critérios de inclusão e exclusão do estudo. Os critérios de inclusão foram: indivíduos que trabalham há mais de 3 anos na função e que executam a atividade por mais de 4 horas ao dia.

A pesquisa foi aprovada pelo Comitê de Ética em pesquisa da Universidade Federal de Santa Catarina sob parecer $\mathrm{n}^{\circ}$ 05372012.9.0000.0121. Todos os participantes assinaram o Termo de Consentimento Livre e Esclarecido.

\subsection{Etapas}

O estudo foi dividido em três etapas:

Levantamento bibliográfico acerca da situação estudada; Coleta de dados in loco; Análise dos dados coletados.

\subsection{Materiais e Métodos}

A coleta de dados foi realizada utilizando a dinamometria de força manual e a captura de 


\section{$16^{\circ}$ \\ ERGODESIGN USIHC CINAHPA}

$16^{\circ}$ Ergodesign - Congresso Internacional de Ergonomia e Usabilidade de Interfaces Humano Tecnológica: Produto, Informações Ambientes Construídos e Transporte

$16^{\circ}$ USIHC - Congresso Internacional de Ergonomia e Usabilidade de Interfaces Humano Computador

CINAHPA | 2017 - Congresso Internacional de Ambientes Hipermídia para Aprendizagem. movimento.

\subsubsection{Captura de Movimento}

A captura de movimentos por sensores inerciais foi utilizada com o objetivo de analisar a frequência dos movimentos, as amplitudes articulares e o tempo de execução da tarefas de desmembramento do cacho.

Esta medida objetiva foi realizada com um equipamento composto por 17 sensores inerciais fixados em diferentes partes do corpo (Xsens MVN Biomech ${ }^{\mathrm{TM}}$, Enschede, The Netherlands) que rastreiam os segmentos, a orientação, a posição, a movimentação e o centro de massa. O sistema funciona em tempo real e a captura é realizada a uma frequência de $120 \mathrm{~Hz}$. Os dados são transmitidos via Wireless para um computador com software que permite a observação, gravação e análise dos movimentos, a partir de gráficos dos ângulos das articulações, da velocidade e duração dos movimentos (Roetenberg, Luinge and Slycke, 2013). Cada sensor contém 3 acelerômetros ortogonais lineares e 3 giroscópios ortogonais (Shippen and May, 2016). Este sistema é confiável e fácil de usar para coleta de dados dentro ou fora de um laboratório (Zhang et al., 2013).

$\mathrm{O}$ equipamento de captura de movimentos por sensores inerciais foi instalado nos trabalhadores e calibrados conforme as orientações do fabricante (Xsens MVN Biomech ${ }^{\mathrm{TM}}$, Enschede, The Netherlands). Posteriormente, os trabalhadores realizaram suas tarefas de maneira que normalmente desempenham por 5 minutos. Assim, os dados obtidos foram analisados no software Xsens MVN Studio Pro e exportados para o software Microsoft Office Excel 2010 para tabulação dos dados e obtenção das médias e os desvios padrão das amplitudes de movimento das articulações e do tempo de execução das tarefas para identificação dos riscos de lesões musculoesqueléticas das tarefas.

\subsubsection{Dinamometria}

Para avaliação da força muscular, utilizou-se o dinamômetro de preensão manual da marca
SAEHAN ® (SAEHAN Corporation, Korea, Modelo DIGI II) com capacidade máxima de $90 \mathrm{~kg}$ e escala de $1 \mathrm{~g}$. No momento dos testes, o indivíduo esteve posicionado conforme recomendação da Sociedade Americana de Terapeutas da Mão (SATM) (SOARES et al., 2012): sentado com os quadris e joelhos a $90^{\circ}$ de flexão, ombro em adução, cotovelo fletido a $90^{\circ}$, antebraço e punho em posição neutra (medioprono). Além disso, o tamanho de empunhadura foi ajustado de acordo com as características antropométricas de cada indivíduo. Iniciando-se com a mão dominante, foram realizadas quatro mensurações em cada lado, sendo a primeira utilizada para adaptação e conhecimento do equipamento e, por conta disso, descartada, com intervalo de 1 minuto entre estas, com intuito de evitar a fadiga muscular. Foi dado verbal foi dado durante o teste e as instruções para sua execução foram padronizadas. Durante as instruções, o volume do comando verbal permaneceu constante para se evitar qualquer influência do mesmo na magnitude da contração muscular (TAYLOR, 2000). Foi utilizada a média dos valores dos três testes de cada mão para a análise dos dados. Este procedimento, recomendado pela SATM (SOARES et al., 2012) e por Figueiredo et al. (2007), é considerada a forma mais confiável de se medir a força de preensão manual. Os dados coletados foram tabulados no Microsoft Office Excel® 2007.

\section{Resultados}

\subsection{Captura do Movimento}

A figura 2 ilustra a postura utilizada na despenca da banana (Takayama et al, 2015) em conjunto com a imagens reais.

Realização:



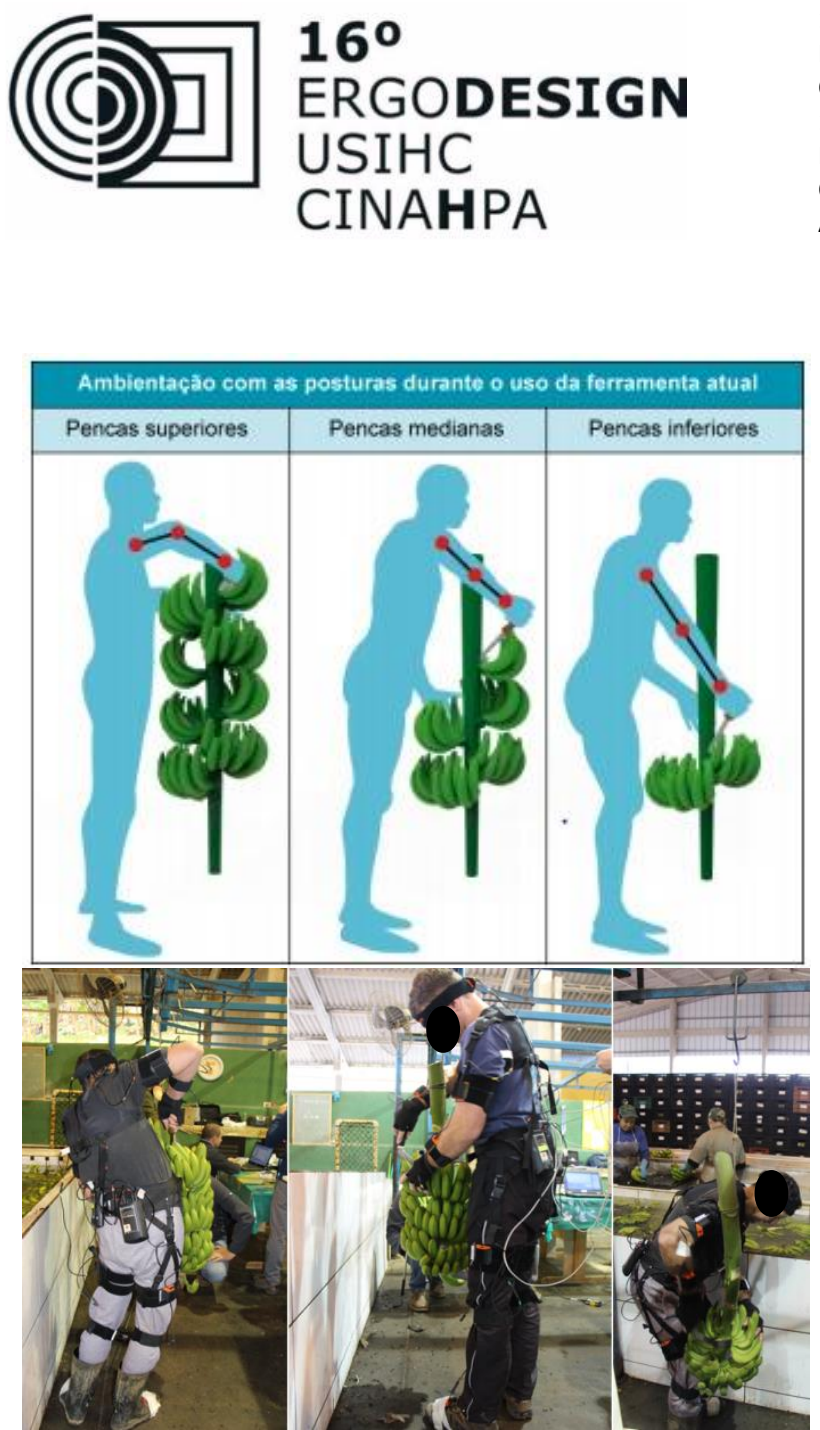

Figura 2: Ilusração de Takayama et al (2015) e imagens reais da atividade (Os Autores, 2017).

A tabela 01 apresenta as médias dos ângulos articulares e os desvios padrão dos trabalhadores durante a tarefa.

\begin{tabular}{|c|c|c|c|c|c|c|}
\hline \multirow[t]{2}{*}{ Articulação } & \multicolumn{6}{|c|}{ Movimento (média em graus) } \\
\hline & Abdução & Adução & $\begin{array}{r}\text { Rotação } \\
\text { medial }\end{array}$ & $\begin{array}{r}\text { Rotação } \\
\text { lateral }\end{array}$ & Flexão & Extensão \\
\hline $\begin{array}{l}\text { Ombro } \\
\text { Direito }\end{array}$ & $\begin{array}{l}32,38 \\
(13,88)\end{array}$ & $\begin{array}{l}1,61 \\
(3,30)\end{array}$ & $\begin{array}{l}28,19 \\
(19,28)\end{array}$ & $\begin{array}{l}11,86 \\
(10,42)\end{array}$ & $\begin{array}{l}33,71 \\
(22,66)\end{array}$ & \begin{tabular}{|l|}
2,31 \\
$(10,76)$
\end{tabular} \\
\hline $\begin{array}{c}\text { Ombro } \\
\text { Esquerdo }\end{array}$ & $\begin{array}{c}23,46 \\
(13,37)\end{array}$ & $\begin{array}{c}2,80 \\
(7,38)\end{array}$ & $\begin{array}{c}17,89 \\
(12,11)\end{array}$ & $\begin{array}{c}18,56 \\
(18,38)\end{array}$ & $\begin{array}{c}28,31 \\
(17,18)\end{array}$ & $\begin{array}{c}4,26 \\
(5,24)\end{array}$ \\
\hline & $\begin{array}{c}\text { Desvio } \\
\text { ulnar }\end{array}$ & $\begin{array}{c}\text { Desvio } \\
\text { radial }\end{array}$ & Pronação & Supinação & Flexão & Extensão \\
\hline $\begin{array}{l}\text { Cotovelo } \\
\text { Direito }\end{array}$ & $\begin{array}{c}4,45 \\
(5,11)\end{array}$ & $\begin{array}{c}27,78 \\
(19,44)\end{array}$ & $\begin{array}{c}77,17 \\
(25,16)\end{array}$ & $\begin{array}{c}0,59 \\
(4,96)\end{array}$ & $\begin{array}{c}52,91 \\
(23,46)\end{array}$ & $\begin{array}{c}0,13 \\
(1,63)\end{array}$ \\
\hline Punho Direito & $\begin{array}{l}10,77 \\
(8,04)\end{array}$ & $\begin{array}{c}11,42 \\
(10,75)\end{array}$ & $\begin{array}{c}9,81 \\
(7,70)\end{array}$ & $\begin{array}{c}20,33 \\
(15,77)\end{array}$ & $\begin{array}{c}12,33 \\
(11,07)\end{array}$ & $\begin{array}{c}9,05 \\
(11,88)\end{array}$ \\
\hline $\begin{array}{l}\text { Cotovelo } \\
\text { Esquerdo }\end{array}$ & $\begin{array}{c}2,18 \\
(2,78)\end{array}$ & $\begin{array}{c}20,86 \\
(18,85)\end{array}$ & $\begin{array}{c}78,62 \\
(12,93)\end{array}$ & 0,00 & $\begin{array}{c}50,60 \\
(22,84)\end{array}$ & $\begin{array}{c}0,76 \\
(2,77)\end{array}$ \\
\hline $\begin{array}{l}\text { Punho } \\
\text { Esquerdo }\end{array}$ & $\begin{array}{l}11,88 \\
(9,29)\end{array}$ & $\begin{array}{c}5,70 \\
(5,01)\end{array}$ & $\begin{array}{c}8,11 \\
(6,67)\end{array}$ & $\begin{array}{c}18,13 \\
(13,35)\end{array}$ & $\begin{array}{l}10,50 \\
(8,96)\end{array}$ & $\begin{array}{c}14,82 \\
(10,44)\end{array}$ \\
\hline
\end{tabular}

Tabela 1: média dos ângulos articulares e os desvios padrão dos trabalhadores durante a tarefa.

\subsection{Dinamometria Isométrica de Preensão Manual}

$16^{\circ}$ Ergodesign - Congresso Internacional de Ergonomia e Usabilidade de Interfaces Humano Tecnológica: Produto, Informações Ambientes Construídos e Transporte

$16^{\circ}$ USIHC - Congresso Internacional de Ergonomia e Usabilidade de Interfaces Humano Computador

CINAHPA | 2017 - Congresso Internacional de Ambientes Hipermídia para Aprendizagem.

A figura 3 ilustra o teste de dinamometria isométrica de preensão manual.

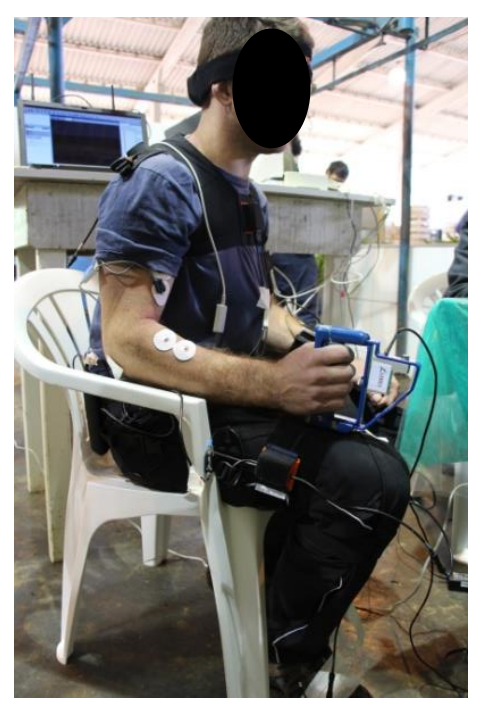

Figura 3: Ilustração do teste de dinamometria isométrica de preensão manual (LABIN-UDESC)

A tabela 2 apresenta os valores das médias do pico de força isométrica de preensão manual de dois trabalhadores da bananicultura. Nessa medida foram avaliados dois indivíduos devido a limitação do tempo de coleta de dados.

\begin{tabular}{cccc}
\hline & Trabalhador l & Trabalhador 2 & Médias \\
\hline $\begin{array}{c}\text { Mão } \\
\text { direita } \\
\text { (Kgf) }\end{array}$ & $67,86 \pm 2,60$ & $41,7 \pm 0,61$ & 54,78 \\
$\begin{array}{c}\text { Mão } \\
\begin{array}{c}\text { esquerda } \\
\text { (Kgf) }\end{array}\end{array}$ & $62,33 \pm 0,32$ & $46,13 \pm 0,40$ & 54,23 \\
\hline
\end{tabular}

Tabela 2: Média dos picos de força de preensão manual em Kgf.

\section{Discussão}

Em relação à análise do movimento, no ombro direito, foi encontrada média dos ângulos de abdução de $32,38^{\circ} \pm 13,88$ e flexão de $33,71^{\circ} \pm$
Realização:
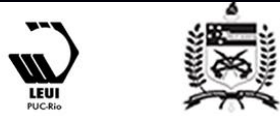


\section{$16^{\circ}$ ERGODESIGN USIHC CINAHPA}

$16^{\circ}$ Ergodesign - Congresso Internacional de Ergonomia e Usabilidade de Interfaces Humano Tecnológica: Produto, Informações Ambientes Construídos e Transporte

$16^{\circ}$ USIHC - Congresso Internacional de Ergonomia e Usabilidade de Interfaces Humano Computador

CINAHPA | 2017 - Congresso Internacional de Ambientes Hipermídia para Aprendizagem.
22,66. Nesse sentido, as posturas adotadas pelo ombro direito poderão ser capazes de gerar lesões osteomusculares, pois diversas pesquisas têm relacionado trabalhos com os ombros em flexão e/ou abdução (Rockwood e Matsen, 1988; Punnett et al., 2000) e flexão e/ou abdução maior que $30^{\circ}$ (Frost e Andersen, 1999). No entanto, outras pesquisas indicam que existe risco de distúrbios musculoesqueléticos com o ombro em flexão e/ou abdução maior que $90^{\circ}$ (Punnett et al., 2000) ou acima de $60^{\circ}$, pois ocorre redução do fluxo sanguíneo e o impacto da musculatura do ombro nas estruturas osteoligamentares (Pope et al., 2001; Stenlund, Lindbeck e Karlsson, 2002; Leclerc et al., 2004).

As posturas dos punhos que apresentaram maiores médias de amplitudes de movimento foram desvio ulnar $\left(10,77^{\circ} \pm 8,04\right)$, desvio radial $\left(11,42^{\circ} \pm\right.$ $10,75)$ e flexão $\left(12,33^{\circ} \pm 11,07\right)$ do punho direito, desvio ulnar $\left(11,88^{\circ} \pm 9,29\right)$, flexão $\left(10,50^{\circ} \pm 8,96\right)$ e extensão $\left(14,82^{\circ} \pm 10,44\right)$ do punho esquerdo. É importante salientar que os três trabalhadores são destros e utilizam a mão esquerda para segurar o cacho de banana, enquanto que a mão direita faz o desmembramento dos cachos de banana com a ferramenta manual. Tendo em vista que as posturas neutras de punho são aquelas que apresentam até $15^{\circ}$ de flexão e extensão (Hedge, 1998), $10^{\circ}$ de desvio ulnar e $15^{\circ}$ de desvio radial (Palmer et al, 1985), verifica-se que durante o desmembramento dos cachos de banana foram encontradas posturas que podem gerar risco de distúrbios musculoesqueléticos nos punhos direito e esquerdo, pois diversas pesquisas têm associado posturas de punho com distúrbios musculoesqueléticos, uma vez que há diminuição da força de preensão em relação a posição neutra quando o punho está em flexão, extensão, desvio ulnar e radial (McGorry, 2001). Enquanto isso, na extensão de punho há aumento de pressão no nervo mediano, na região do punho (Topp e Boyd, 2012) principalmente a partir de $15^{\circ}$ de extensão de punho (Loh e Muraki, 2015).

Apesar do risco demonstrado, inerente ao tipo de movimentação nos membros superiores, a força isométrica de preensão manual obteve valores acima da média nacional para homens (Membro
Dominante: 44,2 $\pm 8,9$ e Membro Não Dominante: 40,5 $\pm 8,5)$ (CAPORRINO, F.A et al, 1998)

\section{Conclusões}

Os resultados da pesquisa mostraram que a atividade da despenca de bananas apresenta riscos de distúrbios osteomusculares no ombro direito, nos punhos direito e esquerdo dos trabalhadores devido às posturas adotadas e a repetitividade.

Em relação à força isométrica de preensão manual, o fato dos indivíduos estudados apresentarem valores acima da média nacional, pode estar relacionado ao fortalecimento inerente à atividade de trabalho.

O método utilizado para realização desse estudo mostrou-se adequado. Sugere-se para o futuro a avaliação eletromiográfica da musculatura dos membros superiores para compreensão mais aprofundada dos riscos nesse segmento.

As conclusões deste estudo contribuem com informações sobre o trabalho agrícola, mais especificamente da atividade de despenca de banana, no que tange sua demanda física, onde há uma lacuna de conhecimentos científicos acerca do comportamento das estruturas musculoesqueléticas e da biomecânica durante a atividade de trabalho. Com dados concretos, intervenções futuras podem ser sugeridas, impactando diretamente na saúde dos trabalhadores, com benefícios para as famílias que laboram na agricultura e para governo por meio da redução de custos com afastamento dessa classe de sua rotina laboral.

Diante dos resultados da pesquisa, verifica-se a necessidade da aplicação dos conhecimentos da ergonomia para propor soluções de baixo custo, para auxiliar a despenca de banana e diminuir o risco de distúrbios osteomusculares nos trabalhadores.

\section{BIBLIOGRAFIA}

CAPORRINO, F.A et al. Estudo populacional da força de preensão palmar com dinamômetro Jamar®. Rev Bras Ortop _ Vol. 33, № 2 - 
$16^{\circ}$ USIHC - Congresso Internacional de Ergonomia e Usabilidade de Interfaces Humano Computador

CINAHPA | 2017 - Congresso Internacional de Ambientes Hipermídia para CINAHPA Aprendizagem.

Fevereiro, 1998.

CULLEN, G. Paisagem Urbana. Lisboa: Edições $70,1996$.

FROST, P., ANDERSEN, J.H., 1999. Shoulder impingement syndrome in relation to shoulder intensive work. Occup. Environ. Med. 56: 494-8.

HEDGE, A., 1998. Design of hand-operated devices. In: Stanton, N. (Ed.), Human Factors in Consumer Products. Taylor \& Francis, London, pp. 203-222.

LECLERC, A., CHASTANG, J.F., NIEDHAMMER, I., LANDRE, M.F., ROQUELAURE, Y., 2004. Incidence of shoulder pain in repetitive work. Occup. Environ. Med. 6, 39-44.

LOH, P.Y., MURAKI, S., 2015. Effect of Wrist Angle on Median Nerve Appearance at the Proximal Carpal Tunnel. PLoS ONE 10(2), e0117930.

MCGORRY, R.W., 2001. A system for the measurement of grip forces and applied moments during hand tool use. Appl. Ergon. 32, 271-278.

PALMER, A.K., WERNER, F.H., MURPHY, D., GLISSON, R., 1985. Functional wrist motion: a biomechanical study. The Journal of Hand Surgery 10A (1),39-46.

POPE, D.P., SILMAN, A.J., CHERRY, N.M., PRITCHARD, C., MACFARLANE, G.J., 2001. Association of occupational physical demands and psychosocial working environment with disabling shoulder pain. Am. Rheum. Dis. 60, 852-858.

PUNNETT, L., FINE, L.J., KEYSERLING, W.M., HERRIN. G.D., CHAFFIN, D.B., 2000. Shoulder disorders and postural stress in automobile assembly work. Scand. J. Work. Environ. Health 26(4), 283-291.

ROCKWOOD, C.A.J., MATSEN III, F.A. The shoulder. Philadelphia: W.B. Saunders Company,
1988.

SOARES, A.V.; JÚNIOR, CARVALHO JUNIOR J.M.; SILVA, L.; BORGES JUNIOR N.G.; DOMENECH, S. C. Valores normativos da dinamometria lombar: um teste funcional para o tronco. Anais do IV Simpósio em

Neuromecânica Aplicada. p. 55-56, 2013.

SOARES, A. V., CARVALHO JUNIOR, J.M.DE, FACHINI, J., DOMENECH, S.C., BORGES JÚNIOR, N.G. Correlação entre os testes de dinamometria de preensão manual, escapular e lombar. Revista Acta Brasileira do Movimento Humano, Ji-Paraná, v. 2, n. 1, p. 65-72, 2012.

STENLUND B, LINDBECK L, KARLSSON D. Significance of house painters' work techniques on shoulder muscle strain during overhead work. Ergonomics 2002, 45, 455-468.

TAKAYAMA, L, MERINO, E.A.D, MERINO, G.S.A.D. Análise postural na pós-colheita da banana: etapa de despencamento. $15^{\circ}$ ERGODESIGN - Congresso Internacional de Ergonomia e Usabilidade de Interfaces Humanotecnologia I $15^{\circ}$ USIHC - Congresso Internacional de Ergonomia e Usabilidade de Interfaces Humano-computador, Blucher Design Proceedings, Volume 2, 2015, Pages 787-798.

TOPP, K.S., BOYD, B.S., 2012. Peripheral nerve: From the microscopic functional unit of the axon to the biomechanically loaded macroscopic structure. Journal of Hand Therapy 25, 142-152.

\section{Agradecimentos}

Os autores agradecem a Coordenação de Aperfeiçoamento de Pessoal de Nível Superior (CAPES), ao Conselho Nacional de Desenvolvimento Científico e Tecnológico (CNPq), a Fundação de Amparo à Pesquisa e Extensão Universitária (FAPEU), a Rede de Pesquisa e Desenvolvimento em Tecnologia Assistiva (RPDTA), Empresa de Pesquisa Agropecuária e Extensão Rural de Santa Catarina (EPAGRI) e ao Núcleo de Gestão em Design e Laboratório de Design Universal da Universidade
Realização:

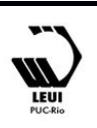


$16^{\circ}$

ERGODESIGN USIHC CINAHPA $16^{\circ}$ Ergodesign - Congresso Internacional de Ergonomia e Usabilidade de Interfaces Humano Tecnológica: Produto, Informações Ambientes Construídos e Transporte

$16^{\circ}$ USIHC - Congresso Internacional de Ergonomia e Usabilidade de Interfaces Humano Computador

CINAHPA | 2017 - Congresso Internacional de Ambientes Hipermídia para Aprendizagem.

Federal de Santa Catarina NGD-LDU/UFSC que viabilizaram esta pesquisa. 